\title{
Book review: Water justice
}

\author{
Thomas Thaler \\ Institut für Alpine Naturgefahren, Universität für Bodenkultur, Wien, Österreich \\ Correspondence: Thomas Thaler (thomas.thaler@boku.ac.at) \\ Published: 19 March 2020
}

Boelens, R., Perreault, T., and Vos, J.: Water Justice, Cambridge University Press, Cambridge, p. 392, ISBN 9781-107-17908-0, EUR 67.43, 2018.

Wasser spielt eine wichtige Rolle in unserer Gesellschaft und deshalb waren und sind Fragen zur Ver- und Entsorgung, zu Gewässerschutz sowie des Zugangs zu Wasser immens wichtig und werden dementsprechend auch kontrovers diskutiert. Im Laufe der Geschichte wurden sie immer wieder neu formuliert und beantwortet. Diese existenziellen Fragen nach sauberem und trinkbarem Wasser, einer funktionierenden Abwasserentsorgung sowie dem Schutz vor extremen hydrologischen Wetterereignissen, wie z.B. Hochwasser und Dürre, werden immer intensiv innerhalb der Gesellschaft diskutiert. Eine erhebliche Problematik betrifft das Eigentumsverhältnis. Soll oder darf die Versorgung öffentlich bzw. privat organisiert werden? Das Streitgespräch, wie das wertvolle Gut Wasser geregelt werden soll, sowie die Auseinandersetzungen, wer davon profitiert bzw. im Gegensatz dazu verliert, findet nicht erst mit der Ausbreitung des Neoliberalismus statt, wurde aber erst in den letzten Jahrzehnten in den wissenschaftlichen Diskussionen wiederentdeckt und stärker untersucht. Insbesondere die Debatte über einen fairen $\mathrm{Zu}$ gang wurde innerhalb der wissenschaftlichen Auseinandersetzung im Bereich der Umweltgerechtigkeit (Environmental Justice), die ihren Ausgang in den Vereinigten Staaten von Amerika in den 1980er Jahren nahm, geführt. Die Thematik über das Recht auf sauberes Trinkwasser sowie das Recht auf Schutz vor Hochwasserereignissen wurde verstärkt diskutiert.

Der Sammelband „Water Justice“, herausgegeben von Rutgerd Boelens, Tom Perreault und Jeroen Vos bei Cambridge University Press, greift die Frage nach einem gerechten Zugang zu Wasser und damit einhergehenden Konflikten auf, die vor allem durch die Herausforderungen des 21. Jahrhunderts und vor allem durch den Klimawandel ge- prägt sind. Es werden auch andere ökonomische und gesellschaftliche Entwicklungen thematisiert, so z.B. Änderungen in der Landnutzung, wie die Entstehung von Megastädten global oder den Wandel innerhalb der Industrie, des Bergbaus sowie der Forst- und Landwirtschaft, der grundsätzlich zukünftig vermehrte Wassernutzung bedingen wird. Folglich werden zukünftig verstärkt bestimmte Personenkreise ihre Interessen auf die vorhandenen verfügbaren Oberflächenund Grundwasserreserven beanspruchen.

Der Band umfasst insgesamt 19 Kapitel, wo anhand von verschiedenen Beispielen rund um den Globus Wasserkonflikte und das Verständnis von Wassergerechtigkeit aufgezeigt werden. Diese beinhalten Exempel der städtischen Trinkwasserversorgung, die Ausschließung von Personengruppen zum Zugang von Grundwasserreserven, Hochwasserschutz bzw. Verschmutzung der Wasserversorgung aufgrund von Schwerindustrie bzw. durch den Austritt von Quecksilber aus Bergwerken. Der Band beinhaltet unterschiedliche kritische theoretische Konzepte aus der politischen Ökologie und kritischen Geografie, wie beispielsweise Konzepte der Hegemonie von Antonio Gramsci bzw. feministische Arbeiten wie das Kapitel 18 von Lisa Bossenbroek und Margreet Zwarteveen, wo die Verteilung von Wasserreserven und -vorräten von ländlichen Gemeinden hin zu landwirtschaftlichen Exportprodukten aus der Sicht feministischer Theoriearbeit untersucht wird. Anhand der Vielzahl unterschiedlicher Fallstudien aus dem Globalen Norden und Süden werden historische sowie zeitgenössische Konflikte und Gerechtigkeitskonzepte dargestellt.

Der Sammelband wird in vier Themenschwerpunkte unterteilt: (1) Re-Politisierung der Wasserversorgung (Kapitel 1 bis 5), (2) Gestaltung der Wasserinfrastruktur (Kapitel 6 bis 9), (3) Konflikte im Partizipationsprozess (Kapitel 10 bis 14), sowie (4) Diskurse und Imaginationen in der Gouvernementalität (Kapitel 15 bis 18).

Der erste Schwerpunkt liegt auf der theoretischen Grundlage der politischen Interventionen im Bereich der Wasser- 
versorgung, wobei der Diskurs um die Wasserpolitik insbesondere in den vergangenen Jahren verstärkt in einem unpolitischen Dialog geführt wurde. Vorrangig die Planung einer nachhaltigen Wasserpolitik beinhaltet zahlreiche Konflikte zwischen den konkurrierenden Vorstellungen und Ideen, wie man den Zugang, die Verteilung und den Schutz in der Wasserwirtschaft organisieren soll. Hier spielt auch die Frage nach der Deutung, wie Wassergerechtigkeit auszusehen hat, eine äußerst wichtige Rolle. Der zweite Abschnitt beschäftigt sich mit den Herausforderungen der Wassergerechtigkeit in Hinblick auf umstrittene Entwicklungsparadigmen und Fortschrittsglauben in der Vergangenheit. Hierbei liegt der Fokus darauf, wie der Staat Markt und Ingenieurswissenschaft, Eingriffe in die Wasserversorgung und Hochwasserschutz für die Gesellschaft nach deren Gesichtspunkt und Deutung gestaltet. Der dritte Abschnitt befasst sich mit den Mechanismen, die angewandt werden, um gezielt bestimmte Menschen von Wasserkonflikten auszuschließen und welche Gegenmaßnahmen (u.a. durch gezielte Zusammenarbeit mit anderen Akteuren*innen) von den betroffenen Personengruppen getroffen werden, um wieder wahrgenommen zu werden in der Beanspruchung ihrer Rechte und Ziele um das Gut Wasser. Der vierte und letzte Abschnitt beschäftigt sich vorwiegend mit den aktuellen Diskursen, wie z.B. virtuelles Wasser oder Konflikte von kulturellen Identitäten, wie beispielsweise die kulturellen Erinnerungen der Menschen im Huanuni-Flusstal auf dem bolivianischen Altiplano, die massiv von Wasserverschmutzungen durch den Bergbau betroffen sind, der nicht immer im Einklang mit dem Schutz der Wasserressource erfolgt war.

In den vier Abschnitten dieses Sammelbands werden insgesamt fünf Konfliktfelder untersucht, nämlich die intensive Nutzung von Wasser in der Landwirtschaft, die im Rahmen des Land Grabbing in den letzten Jahren massiv zugenommen hat. Die Folgen haben wiederum massive Auswirkungen auf die lokale Wasserversorgung (Water Grabbing), die dadurch die Konflikte zwischen der Agrarindustrie und den lokalen Familien massiv verschärft haben. Der zweite Konflikt beschäftigt sich mit der Wasserverschmutzung durch Industrie und Bergbau, wie im Beispiel von Flint, Michigan (Vereinigte Staaten von Amerika). Dort mussten einkommensschwächere Haushalte im Vergleich zur weißen Mittelschicht unter massiven Wasserverschmutzungen leiden. Die dritte Problematik betrifft die Auswirkung der Errichtung von Wasserkraftwerken, die meist in ländlichen, peripheren Regionen unter der Prämisse der nachzuholenden Entwicklung bzw. Rückständigkeit der lokalen Bevölkerung argumentiert wird, wie Beispiele aus der Türkei oder Guatemala zeigen. Des Weiteren werden Konflikte im Hochwasserschutz, die häufig zu einem unterschiedlichen Schutzniveau innerhalb der Gemeinde führen können, betrachtet. Ein vierter zentraler Konflikt besteht in den Herausforderungen mit der Umleitung von Wasser zwischen verschiedenen Wassereinzugsgebieten, ländlichen und städtischen Regionen bzw. innerhalb einer Stadt, die nicht nur zur ungleichen Verteilung führen kann, sondern auch eine massive Auswirkung auf das Ökosystem haben kann. Der letzte Schwerpunkt liegt auf der Privatisierung der Wasserver- und Abwasserentsorgung, wo informelle Siedlungen keine Möglichkeit zu sauberem Wasser oder einem funktionierenden Abwassersystem haben, da die Gebühren für die Nutzung der lokalen Bevölkerung weit über ihrem durchschnittlichen Haushaltseinkommen liegen.

Der Band stützt sich auf umfassende Ansätze der Umweltgerechtigkeit aus der Perspektive der politischen Ökologie, wo Wasser neben einer materiellen Funktion vor allem aus der Vorstellung der Gesellschaft definiert wird und sich dadurch die Konflikte das Wasser betreffend auch über die Jahrhunderte hinweg immer wieder verändert haben und neu formuliert werden mussten. Dabei ändert sich eben auch die Situation der Konflikte, da immer wieder neue Wettbewerber bzw. Entwicklungen, wie z.B. die Industrialisierung der Forst- und Landwirtschaft, Siedlungsentwicklungen etc., um die Ressource Wasser kämpfen. Wassergerechtigkeit schließt die Frage nach der Verteilung ein, berücksichtigt aber auch Fragen der kulturellen Anerkennung und politischen Beteiligung. Hierbei ist ein wichtiger Punkt die Berücksichtigung der Betrachtungsweise des menschlichen Handelns auf das jeweilige Ökosystem in Hinblick auf dessen sozio-politischen Rahmen.

Die große Herausforderung besteht in einer Verbindung der sich stetig verändernden politischen Konzepte und Ideen mit gesellschaftlichen Vorstellungen sowie technologischen Entwicklungen mit einer immer wieder sich ändernden Wassergerechtigkeit. Sokrates bemerkte, dass im antiken Griechenland ,sozial gerecht" über die Machthaber definiert wurde: Was sie als gerecht empfanden, war gerecht. Dieses Verständnis veränderte sich immer wieder im Laufe der Geschichte - bis heute, wo Wassergerechtigkeit immer stärker in Zusammenhang mit der ungleichen Verteilung und Diskriminierung von bestimmten Personengruppen gebracht wird.

Durch die Vielzahl der unterschiedlichen Fallstudien und des empirischen Materials rund um den Globus zeigt der Band nicht nur sehr gut die Pluralität innerhalb des Diskurses, wie man eigentlich Wassergerechtigkeit definieren kann, sondern auch, was das Ziel wäre und wie das Ziel der Wassergerechtigkeit zu erreichen ist. Dabei wird sehr gut dargestellt, dass es eben keine einheitliche Definition gibt und sich diese über die Jahrhunderte hinweg stetig geändert hat bzw. sich in der Zukunft ändern wird. Diese Entwicklung wird auch durch neue wissenschaftliche Fachgebiete und -kenntnisse sowie technologische Entwicklungen gestärkt, wie beispielsweise die aktuelle Debatte in der Klimapolitik im Zusammenhang mit extremen hydrologischen Wetterereignissen belegt. Darüber hinaus haben auch die politischen Diskussionen des Neoliberalismus in den letzten Jahren dazu geführt, dass der Diskurs nach der Nutzung der Ressource Wasser nicht mehr ausschließlich der öffentlichen Hand zugeschrieben wird, sondern eine verstärkte Debatte in der Marktwirtschaft ausgelöst hat; mit all seinen Konsequenzen, Herausforderungen und neuen Konfliktfeldern. 
Der Sammelband stellt einen wichtigen Ansatz zur Diskussion nach Wassergerechtigkeit und eine wichtige Lektüre für die Leser*innen dar, die sich mit den Herausforderungen der Wassergerechtigkeit beschäftigen, wobei insbesondere auch die Widersprüche und Lösungsideen und -vorschläge in diesem Themenfeld sehr gut aufgezeigt werden, da es keine One-Fits-All-Ergebnisse gibt. Dies wird vor allem im Hinblick auf die zukünftigen Auswirkungen des Klimawandels noch viel stärker diskutiert werden müssen. Der Band zeigt nicht nur, dass Konflikte um das Wasser allgegenwärtig sind, sondern auch sehr unterschiedlich sein können, womit auch die Forderungen nach einem gerechten Zugang, Wasserschutz und -verteilung sehr unterschiedlich gesehen, interpretiert und diskutiert werden können. 